\title{
Magnetic soliton confinement and discretization effects arising from macroscopic coherence in a chiral spin soliton lattice
}

\author{
Y. Togawa, ${ }^{1,2,3,4,{ }^{*}}$ T. Koyama, ${ }^{5}$ Y. Nishimori, ${ }^{1}$ Y. Matsumoto, ${ }^{1}$ S. McVitie, ${ }^{3}$ D. McGrouther, ${ }^{3}$ R. L. Stamps ${ }^{3}$ Y. Kousaka, ${ }^{4,6,7}$ \\ J. Akimitsu, ${ }^{4,6,7}$ S. Nishihara, ${ }^{4,7}$ K. Inoue, ${ }^{4,7,8}$ I. G. Bostrem, ${ }^{9}$ Vl. E. Sinitsyn, ${ }^{9}$ A. S. Ovchinnikov, ${ }^{9}$ and J. Kishine ${ }^{4,10}$ \\ ${ }^{1}$ Department of Physics and Electronics, Osaka Prefecture University, 1-2 Gakuencho, Sakai, Osaka 599-8570, Japan \\ ${ }^{2}$ JST, PREST, 4-1-8 Honcho Kawaguchi, Saitama 333-0012, Japan \\ ${ }^{3}$ School of Physics and Astronomy, University of Glasgow, Glasgow G12 8QQ, United Kingdom \\ ${ }^{4}$ Centre for Chiral Science, Hiroshima University, Higashi-Hiroshima, Hiroshima 739-8526, Japan \\ ${ }^{5}$ Department of Materials Science, Osaka Prefecture University, 1-1 Gakuencho, Sakai, Osaka 599-8531, Japan \\ ${ }^{6}$ Department of Physics and Mathematics, Aoyama Gakuin University, Sagamihara, Kanagawa 252-5258, Japan \\ ${ }^{7}$ Graduate School of Science, Hiroshima University, Higashi-Hiroshima, Hiroshima 739-8526, Japan \\ ${ }^{8}$ IAMR, Facility of Science, Hiroshima University, Higashi-Hiroshima, Hiroshima 739-8530, Japan \\ ${ }^{9}$ Institute of Natural Sciences, Ural Federal University, Ekaterinburg, 620083, Russia \\ ${ }^{10}$ Division of Natural and Environmental Sciences, The Open University of Japan, Chiba, 261-8586, Japan \\ (Received 8 June 2015; revised manuscript received 5 October 2015; published 17 December 2015)
}

\begin{abstract}
We present how macroscopically coherent ordering within a chiral state can be manifested in the physical properties within the context of an archetypical system-the chiral spin soliton lattice in a monoaxial chiral magnet $\mathrm{CrNb}_{3} \mathrm{~S}_{6}$. Using magnetotransport measurements and state-of-the-art Lorentz electron microscopy, we demonstrate spin soliton confinement in $1-\mu \mathrm{m}$-wide grains with different crystalline chirality and discretized magnetoresistance in 10- $\mu \mathrm{m}$-wide crystals. Discretization effects are found to be prominent when the system size is reduced to the order of $10 \mu \mathrm{m}$ along the chiral axis. A consequence that we identify is a robust coherence of the chiral soliton lattice against deformation. The spin configuration at the grain boundaries, which leads to soliton confinement, is experimentally clarified.
\end{abstract}

DOI: 10.1103/PhysRevB.92.220412

PACS number(s): 75.25.-j, 68.37.Lp, 75.47.-m, 75.50.Cc

Helicoidal spin systems were among of the first chiral spin systems to be studied [1]. An incommensurate-commensurate (IC-C) phase transition was proposed several decades ago by Dzyaloshinskii for this system [1] and a discommensurate spin structure appears when a magnetic field is applied perpendicular to the chiral axis [1-5]. The discommensurate phase is called a chiral soliton lattice (CSL), and is composed of parallel chains of tightly wound spin "twists." The CSL is distinct from and competes with a "forced" ferromagnetic phase in which all spins align parallel with application of a sufficiently large magnetic field.

Recently, the CSL was directly observed and characterized using Lorentz electron microscopy and small-angle electron scattering experiments [6]. In contrast to topological counterparts found in cubic chiral magnets [7-9], the CSL in monoaxial hexagonal crystals [6] are observed to be robust against effects associated with material defects and thermal fluctuations. The reasons for this robustness are not well understood but appear to be related to the presence of only one chiral axis.

The CSL couples with conduction electrons and gives rise to nontrivial physical properties. In particular, each magnetic soliton in the CSL works as a scattering potential for con-

\footnotetext{
*y-togawa@pe.osakafu-u.ac.jp
}

Published by the American Physical Society under the terms of the Creative Commons Attribution 3.0 License. Further distribution of this work must maintain attribution to the author(s) and the published article's title, journal citation, and DOI. duction electrons with itinerant spins and thus induces magnetoresistance (MR) along the chiral axis. Indeed, a negative interlayer MR was observed in a large single crystal $\mathrm{CrNb}_{3} \mathrm{~S}_{6}$ of several $\mathrm{mm}^{2}$ in area in the $a b$ plane and $200 \mu \mathrm{m}$ in thickness along the $c$ axis [10]. The MR was found to vary smoothly with applied magnetic field strength qualitatively as the soliton density, indicating that the scattering frequency as conduction spins traversing the CSL correlates with the soliton density.

Nonmonotonous changes of the MR and magnetization were observed in noncentrosymmetric $\mathrm{MnSi}$ thin films of less than 30-nm thickness [11]. The helicoidal structure with two soliton $2 \pi$ twists is formed normal to the plane because of a uniaxial anisotropy with hard axis normal to the film. Spin configurations at several field strengths below the critical field were examined using polarized neutron reflectometry and the MR behavior was interpreted as a transformation among helicoidal state with a discrete quantized number of solitons.

In this paper, we give evidences that such soliton confinement and discretization effects of physical properties take place in the monoaxial chiral crystal $\mathrm{CrNb}_{3} \mathrm{~S}_{6}$ with the robust CSL. In particular, we show that the small system size strongly affects the MR by creating stepwise and hysteretic changes. Using a combination of magnetotransport measurements and high resolution Lorentz transmission electron microscopy (TEM), we demonstrate experimentally that the discretized MR appears in $10-\mu$ m-wide $\mathrm{CrNb}_{3} \mathrm{~S}_{6}$ crystals and is due to soliton confinement. We visualize the soliton confinement in grains with a different crystalline chirality (which we refer to as "chirality grains") whose width is on the order of $1 \mu \mathrm{m}$. We find similar confinement effects in micrometer-sized 


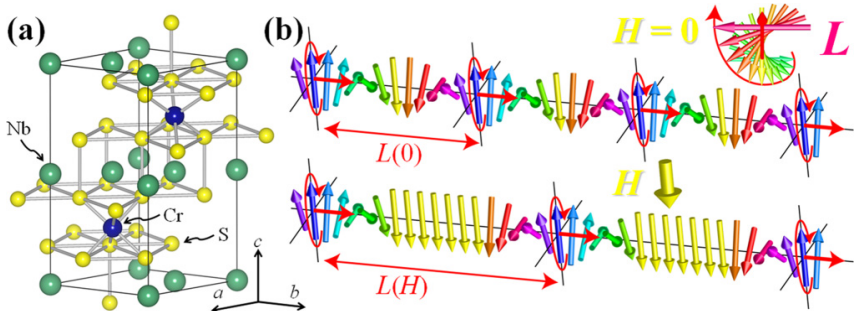

FIG. 1. (Color online) (a) Crystalline structure of a monoaxial chiral crystal $\mathrm{CrNb}_{3} \mathrm{~S}_{6}$. (b) Schematics of chiral spin orderings with left-handed magnetic chirality. Chiral helimagnetic order at zero magnetic field and the CSL in the presence of a magnetic field perpendicular to the chiral axis are represented by colored arrows.

samples and link this directly to stepwise changes in the MR. These properties, as far as we know, have never been observed in bulk single crystals and become apparent only when the sample size is reduced to the order of $10 \mu \mathrm{m}$ or less, i.e., the number of solitons in the system being limited to hundreds or less. These features are consequences of how the spin structure of the CSL remains perfect at least over micrometers.

The system we consider, $\mathrm{CrNb}_{3} \mathrm{~S}_{6}$, is typical of the monoaxial chiral magnets, belonging to the space group of $P_{6} 22$ [15]. It has a layered hexagonal structure of $2 H$-type $\mathrm{NbS}_{2}$ intercalated by $\mathrm{Cr}$ atoms, with the size of unit cell $0.57 \mathrm{~nm}$ in the $a b$ plane and $1.21 \mathrm{~nm}$ along the $c$ axis [16], as shown in Fig. 1(a). The phase transition temperature $T_{\mathrm{C}}$ is found to be $127 \mathrm{~K}$ in the samples examined in this study. In the unit cell, an element block of $\mathrm{Cr}$ atom is surrounded by six neighbor $\mathrm{S}$ atoms and coincides with a neighboring block of the same structure by rotating $\pi$ and sliding a distance $-\boldsymbol{a} / 3+\boldsymbol{b} / 3+\boldsymbol{c} / 2$. As a consequence of combined translational and screw operations, chiral symmetry is guaranteed over all of the crystal and a monoaxial antisymmetric exchange (Dyzaloshinskii-Moriya, subsequently referred to as DM) interaction $[17,18]$ exists all along the chiral $c$ axis.

The formation of the CSL is triggered by the application of a magnetic field, and results from how the spins cope with a competition between Heisenberg exchange (coupling constant $J_{12}$ ), DM antisymmetric exchange interactions and the Zeeman energy. Spin helimagnetic ordering appears at zero magnetic field, as depicted in Fig. 1(b). The magnetic moments rotate in one direction only with respect to the chiral axis which is determined by the DM vector $\boldsymbol{D}$. The helix period $L(0)$ is given by a ratio of two kinds of exchange coupling as $2 \pi a_{0} / \arctan \left(|\boldsymbol{D}| / J_{12}\right) \simeq 2 \pi\left(J_{12} /|\boldsymbol{D}|\right) a_{0}$ with $a_{0}$ being the atomic lattice constant along the chiral axis [1-3]. $\mathrm{In} \mathrm{CrNb}_{3} \mathrm{~S}_{6}$, the ratio $|\boldsymbol{D}| / J_{12}$ is found to be 0.16 , corresponding to $L(0)$ of $48 \mathrm{~nm}[6,15]$.

In magnetic fields perpendicular to the chiral axis, the chiral spin helix is transformed into the CSL; a nonlinear structure comprised of regions with spins aligned parallel to the field periodically separated by chiral soliton $2 \pi$ "kinks." The distribution of angles defining the local spin orientations defines a profile that corresponds to a one-dimensional topological soliton solution of the sine-Gordon equation [1-4]. The period $L(H)$ and density $L(0) / L(H)$ of solitons in the CSL depend upon the magnitude of the perpendicularly applied field.

Micrometer-sized specimens of the monoaxial chiral crystal $\mathrm{CrNb}_{3} \mathrm{~S}_{6}$ were prepared for MR measurements, as shown in Fig. 2(a). Specimens of typically $10 \mu \mathrm{m} \times 10 \mu \mathrm{m} \times 1 \mu \mathrm{m}$ were cut from a bulk single crystal of $\mathrm{CrNb}_{3} \mathrm{~S}_{6}$ and fixed to the Si substrate with patterned gold electrodes by using a focused

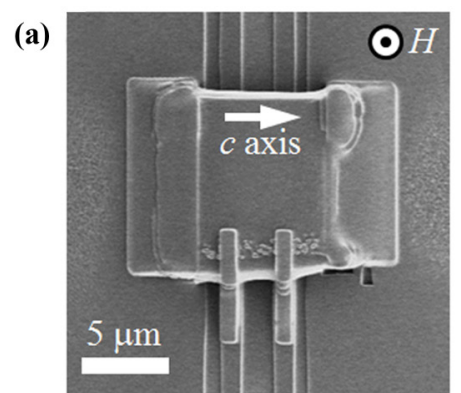

(b)

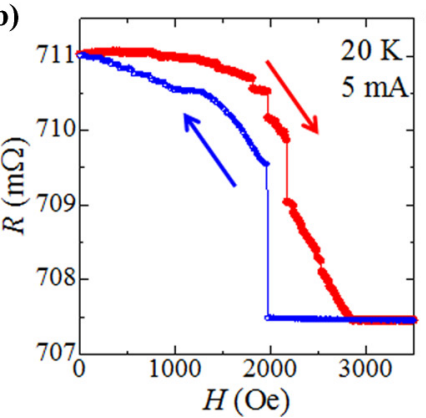

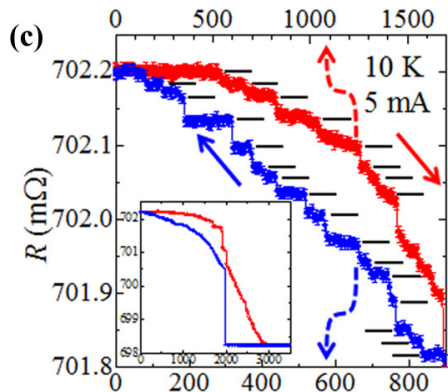

(d) $698.5 \quad H(\mathrm{Oe})$

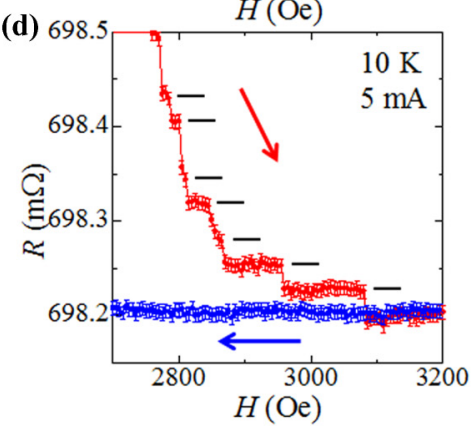

(e)

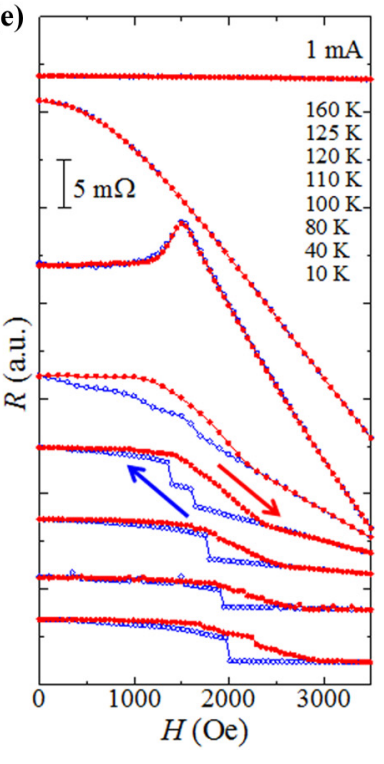

FIG. 2. (Color online) MR data at various temperatures in the micrometer-sized specimen of $\mathrm{CrNb}_{3} \mathrm{~S}_{6}$ crystal. (a) A scanning ion micrograph of the device. MR curves at a temperature of $20 \mathrm{~K}$ (b) and $10 \mathrm{~K}$ (c). The behavior at low and high fields is shown in (c) and (d), respectively, with experimental error bars. (e) The dependence of MR on temperature between 10 and $160 \mathrm{~K}$. The linear dependence above the saturation field was subtracted as the MR background in (b) to (d). 
ion beam technique. The specimen was electrically contacted to four gold electrodes by $\mathrm{W}$ deposition. Two of the electrodes, of $1 \mu \mathrm{m}$ width, were attached to the side and the top surface (ac plane) of the crystal with a typical separation of $1 \mu \mathrm{m}$ for voltage detection, while the latter two were put on the edges for current application. The MR data were taken by the standard four-terminal ac resistance measurement with an ac current amplitude from 0.1 to $5 \mathrm{~mA}$ at a frequency of $137 \mathrm{~Hz}$. A magnetic field was applied perpendicular to the $c$ axis in the plane, and the MR was determined as the field was cycled from zero to 3500 Oe and backward. Measurements were performed in more than ten devices, in all of which similar results were obtained.

With a Lorentz TEM observation [19-21], we visualized the CSL in a thin area (less than $100 \mathrm{~nm}$ ) of $\mathrm{CrNb}_{3} \mathrm{~S}_{6}$ specimens, which had almost the same dimensions in the plane including the $c$ axis as those for the resistance measurements but a thickness smaller by one order of magnitude. Some specimens were found to contain grains with opposite crystalline chirality of $1 \mu \mathrm{m}$ or longer along the $c$ axis. We were able to observe in these grains soliton confinement and discretization. A TEM specimen was mounted on the specimen cooling holder and cooled to below $T_{\mathrm{C}}$. The magnetic field was applied vertically to the specimen by the objective pole-piece by controlling the objective lens current in the $200-\mathrm{kV}$ scanning transmission electron microscope (JEOL ARM-200CF [22]).

First, let us see the MR behavior. Hysteresis is found clearly at low temperatures, as shown in Figs. 2(b) to 2(e), e.g., for measurements at a temperature of $20 \mathrm{~K}$ in Fig. 2(b). Here, the resistance $R$ is given as a function of the field for the increasing (red) and decreasing (blue) branches. $R$ returns to its initial value at zero field but the path for increasing field is different from the path as the field is decreased. A gradual decrease of $R$ is observed with increasing field. Sharp steps appear at 1810 , 1965 , and 2165 Oe on the increasing branch, followed by a cascade of steps with a quasilinear decrease toward a minimum $R$ value at a saturation field $H_{\text {sat }}$ of 2860 Oe. Upon decreasing the field, $R$ remains constant until just below 1965 Oe, when a sudden jump occurs $\left(H_{\text {jump }}\right) . R$ then increases to the initial zero-field value as the field is reduced further. With the field applied along the plane and perpendicular to the $c$ axis, $H_{\text {sat }}$ and $H_{\text {jump }}$ reduce to about 1900 and 800 Oe, respectively (e.g., see the data of the different specimen in Fig. S1 in Ref. [23]). In this case, the value of $H_{\text {sat }}$ is almost consistent with that measured in a configuration of the least demagnetization field [10].

Another feature seen in the MR curve at low and high fields at $10 \mathrm{~K}$ is shown in Figs. 2(c) and 2(d). Many steps are visible in the increasing and decreasing branches in Fig. 2(c). The steps appear with many different plateau widths and the magnitude of the jumps varies between 16 and $2060 \mu \Omega$. Interestingly, seven steps, counted from zero field along the increasing and decreasing field branches, appear at almost the same value of $R$ irrespective of the swept direction of the field. Stepwise structures appear at higher fields close to $H_{\text {sat }}$ along the increasing field branch, as shown in Fig. 2(d). We note that the plateaus become quite wide near $H_{\text {sat }}$ and the magnitude of the jump is integer multiples of $26 \pm 7 \mu \Omega$.

MR curves for temperatures between 10 and $160 \mathrm{~K}$ are shown in Fig. 2(e). The hysteresis in $R$ appears for a wide temperature range below $T_{\mathrm{C}}$ and becomes even broader with decreasing temperature. The hysteresis disappears entirely for fields larger than $H_{\text {sat }}$ as well as when the temperature is increased very close to $T_{\mathrm{C}}$ (e.g., see the MR curve at $120 \mathrm{~K}$ ). The hysteretic behavior observed in a $10-\mu \mathrm{m}$-sized $\mathrm{CrNb}_{3} \mathrm{~S}_{6}$ specimen contrasts with that found in a bulk specimen of $200-\mu \mathrm{m}$ thickness along the $c$ axis, in which no hysteresis is found over all ranges of temperature [10]. The MR varies smoothly for bulk $\mathrm{CrNb}_{3} \mathrm{~S}_{6}$ in accord with the calculated soliton density [10]. The soliton density plays the role of the order parameter for the continuous IC-C phase transition [1-6] associated with the formation of the CSL. This means that each soliton creates a spin-dependent scattering potential for itinerant spins. At low temperatures where the path lengths are long, MR should involve scattering from all solitons and might therefore be used to measure the soliton density. Note that this would also require perfect registry of solitons across the CSL. Indeed, at temperatures below $110 \mathrm{~K}$, a fine structure appears in the form of a sequence of steps along a portion of the increasing and decreasing field branches in Fig. 2(e), suggestive that discrete changes in soliton density are responsible.

We then performed Lorentz TEM, as sketched in Fig. 3(a), in order to understand the microscopic nature of the steps and plateaus observed in the MR curves. Evidence of multiple states of the CSL is observed directly within a grain of reversed crystalline chirality. The spin configuration in the region containing the grain was experimentally determined from analyses of high-resolution differential phase contrast (DPC) images [20,22] as shown in Figs. 3(b) to 3(d). The schematic illustration obtained is given in Fig. 3(e).

A magnetic field applied perpendicular to the plane has the counterintuitive effect of orienting spin moments in plane near the grain boundary, i.e., perpendicular to the applied field direction. Two $\pi / 2$ twists connect these spins with spins on either side of the boundary. The spin configuration of $\pi / 2$ twist from the boundary looks like the surface twist structure at free boundaries found in MnSi thin films [11,12]. Lorentz images (Fig. S2 in Ref. [23]) reveal that this spin configuration remains the same even at fields above $H_{\text {sat }}$, which means that the net direction of in-plane magnetic moments is fixed around the boundary throughout the field cycle described below.

The mechanism of soliton confinement was discussed theoretically in a finite-size chiral system without or with pinning at both boundaries, i.e., free [11,12] or fixed [13] boundary conditions. The influence of dipolar interactions was argued in a nonchiral spin system [14]. From the Lorentz TEM data, it is hard to identify dominant interactions to give preference to any of the confinement mechanisms. Nevertheless, it is naively expected that the existence of another such boundary some distance away, as seen in Fig. 3, creates a confining well for solitons trapped between the two interfaces.

Indeed, stepwise changes of the soliton period in this confining well were observed when the magnitude of the applied magnetic field was changed. We note that great care with the imaging and analyses was taken in order to maintain a fixed position with respect to the grain while changing the field, thereby ensuring that there were no artefacts associated with changes in magnification, e.g., as shown in Figs. 4(a) to 4(c). 


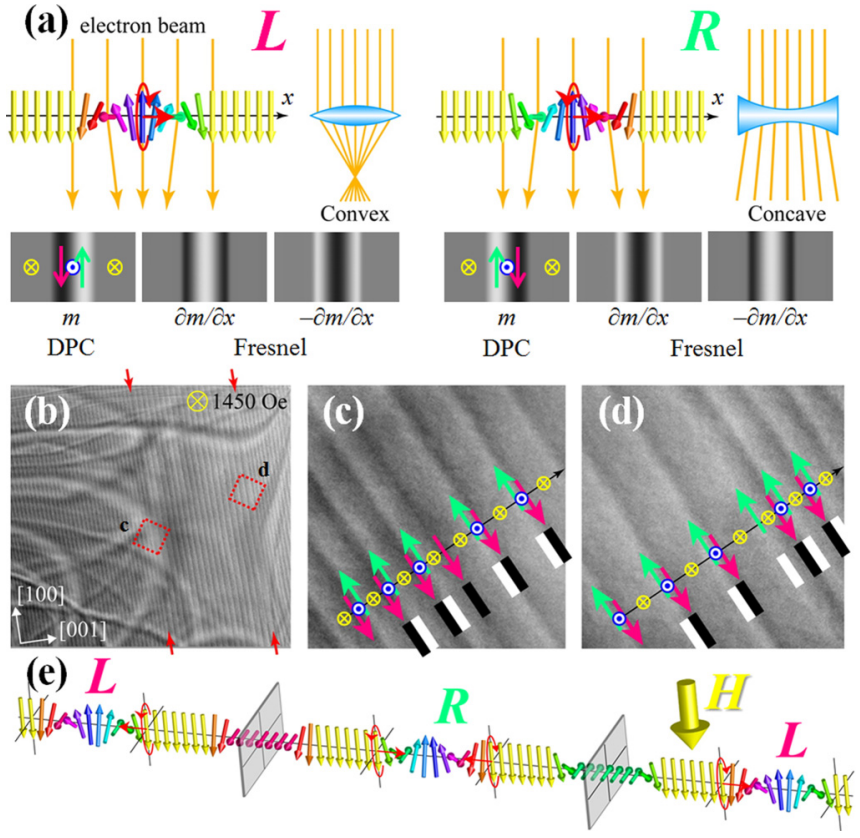

FIG. 3. (Color online) (a) A ray diagram illustrating the relation of the contrast formed in Lorentz micrographs to the chiral soliton twist. The direction of the magnetization $m$ transverse to the electron probe beam is determined from the light and dark contrast. The intensity profile map of in-plane $m$ and its spatial gradient $\partial m / \partial x$ along the chiral axis $x$ correspond closely to micrographs of the soliton taken in the DPC and Fresnel modes, respectively, since the configuration is close to being divergence free. The contrast reverses when we defocus above and below the specimen in the Fresnel mode; $-\partial m / \partial x$ is observed in the underfocused condition. A soliton in the CSL deflects the electron beam in a manner depending on its magnetic chirality, being reminiscent of the cylindrical lenses effect for the optical light. (b) to (d) DPC micrographs of the CSL in a narrow crystal grain of right-handed magnetic chirality sandwiched by wide regions of left-handed magnetic chirality in 1450 Oe at $100 \mathrm{~K}$. The grain boundaries are indicated by arrows in (b). The contrast and direction of $m$ are schematically presented in (c) and (d). (e) Schematics of the spin configuration of the CSL around boundaries where the magnetic chirality switches and solitons could be confined.

Evidence for confinement effect is seen in Figs. 4(d) and 4(e). Plateaus and stepwise jumps are clearly observed for field strength changes of 1 Oe. Note that the spacing between contrast lines within the grain remains constant independent of the magnetic field while within a single plateau range (within experimental resolution). We therefore conclude that the solitons in the right handed region are indeed confined.

Clear and unambiguous evidence for discrete changes in soliton number has been obtained in the present study. We are able to determine exact changes in the soliton numbers in the micro-sized grain discussed here, and argue that the soliton confinement is occurring at the edges of the grain where the in-plane spin configuration is formed. Consequently, the soliton period is discretized in size and the related physical properties are quantized in units of single solitons.

The original prediction of soliton confinement was for a very limited number of solitons in the chiral spin system [11-13]. The difference in soliton numbers between plateaus
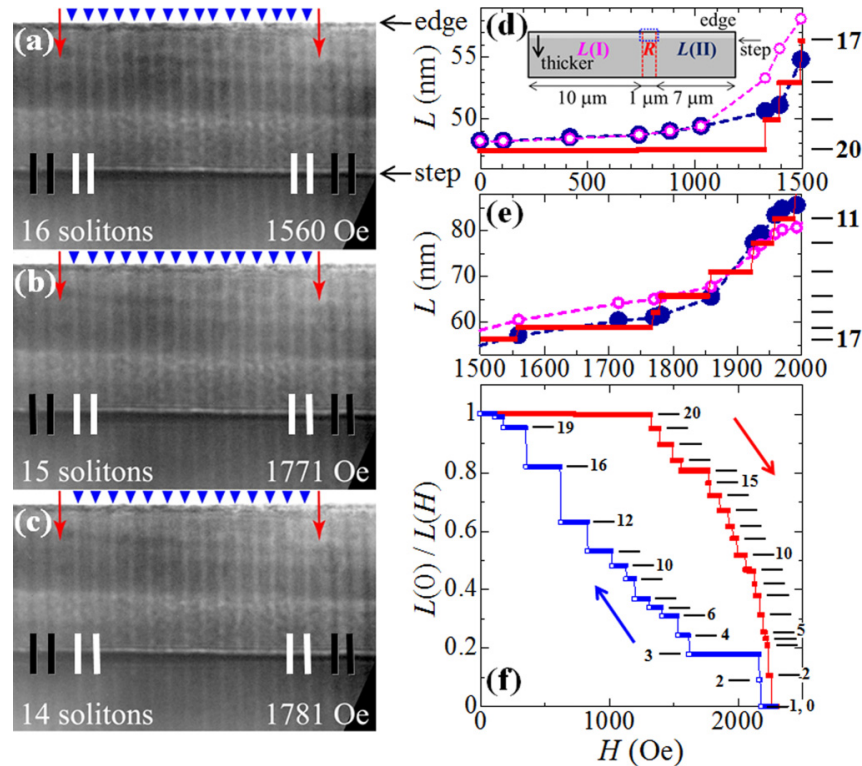

FIG. 4. (Color online) Fresnel micrographs of CSL, taken in the underfocused condition, around a 1- $\mu$ m-width crystal grain of righthanded magnetic chirality at (a) 1560, (b) 1771, and (c) $1781 \mathrm{Oe}$ at $100 \mathrm{~K}$. The chiral boundaries and right-handed solitons confined are indicated by red and blue arrow heads, respectively. The solitons confined reduce in numbers one by one at a particular field with increasing field. (d) and (e) The soliton period as a function of the field, which is measured in a series of Fresnel images monitored at every step of 1 Oe during a cycle of a magnetic field up to the saturation field. The inset shows the sample dimensions. Data taken in the right-handed grain are given in red color, while magenta and dark blue are used to exhibit data in the left-handed grains on the left and right, respectively. (f) The corresponding soliton density. The total number of solitons confined in the right-handed grain is given in (d) to (f). The initial number of confined solitons is 20 .

will increase as the confinement region and number of solitons involved becomes smaller, which enables the detection of MR or magnetization anomalies in a $\mathrm{MnSi}$ thin film associated with the nucleation or annihilation processes of two soliton twists [11]. In this connection, we found that, for specimens on macroscopic length scales, namely in which tens or hundreds solitons are involved in the confinement, we are able to distinguish stepwise changes due to single soliton events along the transition to the forced ferromagnetic state. Indeed, 20 solitons are confined in the case of the chiral grain shown in Figs. 4(d) to 4(f) and about 200 solitons are involved for MR data in Figs. 2(c) and 2(d). We stress that solitons strongly correlate over ten micrometers as observed in Lorentz micrographs and small-angle electron scattering data [6], which is consistent with the present findings.

We expect that in an infinite soliton lattice, the period outside the confined region should change continuously. Outside the grain, the spacing indeed varies continuously with the field strength, for the case when 20 or 16 solitons are found in the right-handed grain. However, a sudden increase of the slope is also frequently observed outwith the grain, e.g., as seen at around 1300 and 1900 Oe in Figs. 4(d) and 4(e). This is consistent with the fact that the soliton lattice is not infinite outside the grain, and is confined over a distance on the 
order of the macroscopic sample size. The confinement effect occurring outwith the grain, namely between the chiral well and a free edge of the specimen, is over a length scale of 10 and $7 \mu \mathrm{m}$.

The length scales over which distinct plateaus are observed are striking. This requires a high degree of coherence of the CSL in order that the CSL at the separate edges works cooperatively to allow a single soliton escape or injection. The confinement mechanism in operation for the $1-\mu \mathrm{m}$ grain confinement probably differs from the one that must occur at free edges, as observed in the $10-\mu \mathrm{m}$ samples, but nevertheless confinement occurs in each situation. Single soliton changes were observed even in finite size MR samples at high fields where the soliton density is smallest. This is consistent with single soliton decrease in the chirality grain throughout the increasing field branch. However, the spacing of the CSL becomes slightly irregular at high fields close to $H_{\text {sat }}$, although solitons are very straight perpendicular to the $c$ axis. The coherence of the CSL may begin to deteriorate at smaller soliton densities partly because of random pinning and other factors, which may be not negligible.

We note an interesting analogy to the notion of generalized thermodynamic rigidity as associated with long range order. In some systems, the rigidity is characterized with reference to a phase, as in the cases of superfluidity and superconductivity. The magnetic order parameter of the CSL is represented by $M_{0} \exp ( \pm i \phi(r))$ where the sign defines the chirality of the state. Vortices in the superconducting or superfluid states are likewise described by an order parameter analogous to $\phi(\boldsymbol{r})$. A quantization of the trapped magnetic flux, superfluid circulation, or the spin soliton lattice period arises from the uniqueness of a wave function and rigidity of an order parameter. In all cases, this requires phase coherence over some characteristic length scale.

In summary, we have studied the domain structure of the CSL at chirality grain boundaries in thin films of $\mathrm{CrN}_{3} \mathrm{~S}_{6}$. As a consequence, we observe microscopic quantization effects for the total number of solitons within the chirality grains and the MR in microsized $\mathrm{CrN}_{3} \mathrm{~S}_{6}$ specimens. The CSL with long-range coherence are so far found solely in a particular class of the monoaxial chiral magnets but could be realized and functioned in other chiral systems such as chiral nematic liquid crystals. In tetragonal, hexagonal, and trigonal systems, there is only one principal crystal axis and consequently the crystal symmetry picks up only one helical axis. These three crystal classes can support a robust CSL structure.

From a technological viewpoint, confinement discretizes the soliton density, thereby enabling the use of solitons as individual and countable objects. Practically, counting the soliton number electrically can be accomplished with relative ease, which allows us to exploit the discreteness of the soliton density for data storage applications.

We thank D. MacLaren and R. Camley for interesting and useful discussions, and acknowledge support from the JSPS Grant-in-Aid for Scientific Research (S) (No. 25220803), JSPS Brain Circulation Project (R2507), JSPS Core-to-Core Program "Advanced Research Networks," the MEXT program for promoting the enhancement of research universities (Hiroshima University), EPSRC EP/M024423/1, the Program of the Ministry of Education and Science of the Russian Federation 02.A03.21.0006 on 27.08.2013, and the Grant RFBR 14-02 92104.

In addition, the open access data link is provided in Ref. [24].
[1] I. E. Dzyaloshinskii, Theory of helicoidal structures in antiferromagnets. I. Nonmetals, Sov. Phys. JETP 19, 960 (1964).

[2] Y. A. Izyumov, Modulated, or long-periodic, magnetic structures of crystals, Sov. Phys. Usp. 27, 845 (1984).

[3] J. Kishine, K. Inoue, and Y. Yoshida, Synthesis, structure and magnetic properties of chiral molecule-based magnets, Prog. Theor. Phys. Suppl. 159, 82 (2005).

[4] P.-G. De Gennes, Calcul de la distorsion d'une structure cholesterique par un champ magnetique, Solid State Commun. 6, 163 (1968).

[5] F. Lonberg and R. B. Meyer, New Ground State for the SplayFréedericksz Transition in a Polymer Nematic Liquid Crystal, Phys. Rev. Lett. 55, 718 (1985).

[6] Y. Togawa, T. Koyama, K. Takayanagi, S. Mori, Y. Kousaka, J. Akimitsu, S. Nishihara, K. Inoue, A. S. Ovchinnikov, and J. Kishine, Chiral Magnetic Soliton Lattice on a Chiral Helimagnet, Phys. Rev. Lett. 108, 107202 (2012).

[7] Y. Ishikawa, K. Tajima, D. Bloch, and M. Roth, Helical spin structure in manganese silicide MnSi, Solid State Commun. 19, 525 (1976)

[8] S. V. Grigoriev, V. A. Dyadkin, D. Menzel, J. Schoenes, Yu. O. Chetverikov, A. I. Okorokov, H. Eckerlebe, and S. V. Maleyev, Magnetic structure of $\mathrm{Fe}_{1-x} \mathrm{Co}_{x} \mathrm{Si}$ in a magnetic field studied via small-angle polarized neutron diffraction, Phys. Rev. B 76, 224424 (2007).

[9] A. Zheludev, G. Shirane, Y. Sasago, N. Koide, and K. Uchinokura, Spiral phase and spin waves in the quasi-twodimensional antiferromagnet $\mathrm{Ba}_{2} \mathrm{CuGe}_{2} \mathrm{O}_{7}$, Phys. Rev. B 54, 15163 (1996).

[10] Y. Togawa, Y. Kousaka, S. Nishihara, K. Inoue, J. Akimitsu, A. S. Ovchinnikov, and J. Kishine, Interlayer Magnetoresistance due to Chiral Soliton Lattice Formation in Hexagonal Chiral Magnet $\mathrm{CrNb}_{3} \mathrm{~S}_{6}$, Phys. Rev. Lett. 111, 197204 (2013).

[11] M. N. Wilson, E. A. Karhu, D. P. Lake, A. S. Quigley, S. Meynell, A. N. Bogdanov, H. Fritzsche, U. K. Rößler, and T. L. Monchesky, Discrete helicoidal states in chiral magnetic thin films, Phys. Rev. B 88, 214420 (2013).

[12] S. A. Meynell, M. N. Wilson, H. Fritzsche, A. N. Bogdanov, and T. L. Monchesky, Surface twist instabilities and skyrmion states in chiral ferromagnets, Phys. Rev. B 90, 014406 (2014).

[13] J. I. Kishine, I. G. Bostrem, A. S. Ovchinnikov, and Vl. E. Sinitsyn, Topological magnetization jumps in a confined chiral soliton lattice, Phys. Rev. B 89, 014419 (2014).

[14] E. Y. Vedmedenko and D. Altwein, Topologically Protected Magnetic Helix for All-Spin-Based Applications, Phys. Rev. Lett. 112, 017206 (2014). 
[15] T. Moriya and T. Miyadai, Evidence for the helical spin structure due to antisymmetric exchange interaction in $\mathrm{Cr}_{1 / 3} \mathrm{NbS}_{2}$, Solid State Commun. 42, 209 (1982).

[16] T. Miyadai, K. Kikuchi, H. Kondo, S. Sakka, M. Arai, and Y. Ishikawa, Magnetic properties of $\mathrm{Cr}_{1 / 3} \mathrm{NbS}_{2}$, J. Phys. Soc. Jpn. 52, 1394 (1983).

[17] I. E. Dzyaloshinskii, A thermodynamic theory of "weak" ferromagnetism of antiferromagnetics, J. Phys. Chem. Solids 4, 241 (1958).

[18] T. Moriya, Anisotropic superexchange interaction and weak ferromagnetism, Phys. Rev. 120, 91 (1960).

[19] P. J. Grundy and R. S. Tebble, Lorentz electron microscopy, Adv. Phys. 17, 153 (1968).
[20] J. N. Chapman and M. R. Scheinfein, Transmission electron microscopies of magnetic microstructures, J. Magn. Magn. Mater. 200, 729 (1999).

[21] Y. Togawa, Small-angle electron scattering of magnetic fine structures, Microscopy 62, S75 (2013).

[22] S. McVitie, D. McGrouther, S. McFadzean, D. A. MacLaren, K. J. O'Shea, and M. J. Benitez, Aberration corrected Lorentz scanning transmission electron microscopy, Ultramicroscopy 152, 57 (2015).

[23] See Supplemental Material at http://link.aps.org/supplemental/ 10.1103/PhysRevB.92.220412 for MR data. Lorentz micrograph at above the saturation field and the system size dependence of soliton confinement are also provided.

[24] Lorentz micrographs in Figs. 3 and 4 are also available at http://dx.doi.org/10.5525/gla.researchdata.234. 